\title{
OUTPUT FEEDBACK NONLINEAR PREDICTIVE CONTROL -A SEPARATION PRINCIPLE APPROACH
}

\author{
Rolf Findeisen ${ }^{*}$ Lars Imsland ${ }^{* *}$ Frank Allgöwer ${ }^{*}$ Bjarne A. Foss ${ }^{* *}$ \\ * Institute for Systems Theory in Engineering, University of Stuttgart, \\ 70550 Stuttgart, Germany, \{findeise,allgower $\} @ i s t . u n i$-stuttgart.de \\ ** Department of Engineering Cybernetics, Norwegian University of \\ Science and Technology, 7491 Trondheim, Norway, \\ $\{$ Lars.Imsland,Bjarne.Foss $\} @ i t k . n t n u . n o$
}

\begin{abstract}
Over the recent years many advances in the area of nonlinear model predictive control have been made. However, with respect to the output feedback problem and predictive control only a limited number of results are available. Most of the existing approaches do only guarantee local stability. In this note we propose the use of a high gain observer in combination with a sampled nonlinear predictive controller for a special MIMO system class. The resulting output feedback leads to semiglobal practical stability and recovery of performance of the state feedback controller. The results are valid for a wide class of NMPC schemes. Thus they can be considered as a special separation principle for NMPC.
\end{abstract}

Keywords: output feedback, nonlinear predictive control, separation principle, semi-global practical stability

\section{INTRODUCTION}

Model predictive control (MPC) for systems described by nonlinear ODEs (shortly NMPC) has received considerable attention over the past years. Several schemes that guarantee stability if full state information is available exist, see for example (Mayne et al. 2000, Allgöwer et al. 1999) for recent reviews.

Fewer results are available in the case when not all states are directly measured. In (Michalska and Mayne 1995) an optimization based moving horizon observer is designed, that together with the NMPC scheme proposed in (Michalska and Mayne 1993) is shown to lead to (semiglobal) closed loop stability. In (Magni et al. 1998, Magni et al. 2001), see also (Scokaert et al. 1997), local asymptotic stability for observer based discrete-time nonlinear MPC for "weakly detectable" systems is obtained. However, these results are of local nature, i.e. stability is guaranteed only for a sufficiently small observer error and no exact information about the required "level of smallness" is available.

To overcome this problem we follow along the lines of "separations principles" for nonlinear systems (Teel and Praly 1995, Atassi and Khalil 1999) and propose the use of high gain observers in conjunction with NMPC. In comparison to (Imsland et al. 2001b) we derive results for the sampled case, i.e. we do not assume that the state feedback is continuously recalculated. Instead we apply open loop control inputs over the sampling intervals which are recalculated at the sampling instances. We show that the resulting control scheme does lead to semiglobal practical stability of the closed loop. We also show that the output feedback control scheme allows for recovery of performance of the state feedback controller if the observer gain is increased and the sampling time is decreased sufficiently. With recovery of performance we mean recovery of the region of attraction and that the rate of convergence of the output feedback scheme approaches that of the state feedback scheme. The results are valid for a wide class of stabilizing NMPC schemes. Thus for the considered system class the result can be seen as a special separation principle for NMPC.

The key ideas are based on recent investigations of the output feedback control problem for nonlinear systems. Based on the output feedback results for fully input-output linearizable systems (Esfandiari and Khalil 1992) different versions of the so called nonlinear separation principle for a rather wide class of systems have been established (Teel and Praly 1995, Atassi and Khalil 1999, Shim and Teel 2001, Maggiore and Passino 2000). Our result is related to the re- 
sults of (Teel and Praly 1995, Atassi and Khalil 1999) in the sense that we also use a high gain observer for the state recovery and that we use the same key steps in the proof. However the consideration of a sampled control scheme makes some inherent changes necessary.

This note is structured as follows: In Section 2 we briefly state the considered system class. Section 3 contains a description of NMPC state feedback and the high gain observer used. In Section 4 we derive the results on the recovery of the state feedback performance and on semiglobal practical stability of the closed loop.

In the following blockdiag $\left(A_{1}, \ldots, A_{r}\right)$ denotes a block diagonal matrix with the matrices $A_{1}, \ldots, A_{r}$ on the "diagonal", while $\operatorname{diag}\left(\alpha_{1}, \ldots, \alpha_{r}\right)$ denotes a diagonal matrix with the scalars $\alpha_{1}, \ldots, \alpha_{r}$ on the diagonal.

\section{SYSTEM CLASS}

We consider continuous time nonlinear systems of the following form:

$$
\begin{aligned}
& \dot{x}=A x+B \phi(x, u) \\
& y=C x .
\end{aligned}
$$

$x \in X \subseteq \mathbb{R}^{n}$ are the system states, the control input is $u \in \overline{\mathcal{U}} \subset \mathbb{R}^{p}$ and the measured output $y \in \mathbb{R}^{p}$. We consider the control of (1) under bounded control action and state constraints, i.e. the system input is required to lie in the set $\mathcal{U}$ and the system states must lie in the set $X$ with:

Assumption $1 \mathcal{U} \subset \mathbb{R}^{p}$ is compact, $\chi \subseteq \mathbb{R}^{n}$ is connected and $(0,0) \in \mathcal{X} \times \mathcal{U}$.

The matrix $A, B$ and $C$ are given by:

$$
\begin{aligned}
& A=\operatorname{blockdiag}\left[A_{1}, \ldots A_{p}\right], A_{i}=\left[\begin{array}{cccc}
0 & 1 & \cdots & 0 \\
0 & 0 & \cdots & 0 \\
\vdots & & & \vdots \\
0 & \cdots & 0 & 0 \\
0 & \cdots & \cdots & 0
\end{array}\right]_{r_{i} \times r_{i}} \\
& B=\operatorname{blockdiag}\left[B_{1}, \ldots, B_{p}\right], B_{i}=\left[\begin{array}{llll}
0 & \cdots & 0 & 1
\end{array}\right]_{r_{i} \times 1}^{\top} \\
& C=\operatorname{blockdiag}\left[C_{1}, \ldots, C_{p}\right], C_{i}=\left[\begin{array}{llll}
1 & 0 & \cdots & 0
\end{array}\right]_{1 \times r_{i}},
\end{aligned}
$$

i.e. the dynamics consists of $p$ integrator chains, with $n=r_{1}+\cdots+r_{p}$. Furthermore we assume, that the following holds:

Assumption 2 The function $\phi: \mathbb{R}^{n} \times \mathcal{U} \rightarrow \mathbb{R}^{p}$ is assumed to be locally Lipschitz in its arguments over the domain of interest with $\phi(0,0)=0, \phi(x, u)$ is well defined for all $x \in \mathbb{R}^{n}$ and $u \in \mathcal{U}^{1}$ and $\phi$ is globally bounded.

The considered system class has an (invertible) observability map that does not depend on the input. The typical source of systems of this class, is an input affine nonlinear system

$$
\dot{x}=f(x)+g(x) u, \quad y=h(x)
$$

with full (vector) relative degree $\left(r_{1}, r_{2}, \ldots, r_{p}\right)$, i.e., $\sum_{i=1}^{p} r_{i}=n$. Then, it is possible to find a coordinate transformation (Isidori 1995) such that the system is described by (1) with $\phi(x, u)$ linear in $u$.

Remark 2.1 In the SISO case, our approach could be expanded to systems of the general form

$$
\dot{x}=f(x, u), y=h(x)
$$

1 This is necessary, since the observer estimate can leave the set $\mathcal{X}$ with $(y, u) \in \mathbb{R}^{2}$ if the observability map $\Psi$

$$
Y=\Psi\left(x, u, \dot{u}, \ddot{u}, \ldots, u^{\left(q_{u}\right)}\right) .
$$

with $Y=\left[y, \dot{y}, \ldots, y^{q_{y}}\right]^{\top}$ is known and invertible for $x$. Then one would have to know the derivatives of $y$ and $u$ to recover the state. These can be easily obtained by adding a chain of integrators to the input and using a high gain observer to recover the derivatives of $y$ and $u$ (Teel and Praly 1994). However, then we must assume that the state feedback controller stabilizes the expanded system. The original constraints on the inputs become state constraints and additional constraints on the $q_{u}$ th derivative of the input have to be added to guarantee compactness of the feasible set of "new" inputs.

\section{SETUP}

The output control feedback scheme is given by a nonlinear model predictive state feedback and a high gain observer for recovery of the system states.

\subsection{State feedback NMPC}

In the framework of predictive control, the input is defined by the solution of an open loop optimal control problem that is solved at sampling instants. For simplicity we denote the sampling instants by $t_{i}$, with $t_{i}-t_{i-1}=\delta$ being the sampling time. For a given $t, t_{i}$ should be taken as the nearest sampling time $t_{i}<t$. $(\cdot)_{i}$ denotes the value of the corresponding variable at the sampling instance $i$, i.e. $x_{i}=x\left(t_{i}\right)$. The open loop optimal control problem solved at any $t_{i}$ is given by:

\section{NMPC open loop optimal control problem:}

$$
\begin{array}{lll}
\text { Solve } \min _{\bar{u}(\cdot)} J\left(\bar{u}(\cdot) ; x_{i}\right) & \\
\text { subject to: } & \overline{\bar{x}}=A \bar{x}+B \phi(\bar{x}, \bar{u}) \quad \bar{x}(\tau=0) \Rightarrow x \\
& \bar{u}(\tau) \in \mathcal{U}, \bar{x}(\tau) \in X \quad \tau \in\left[0, T_{p}\right] \\
& \bar{x}\left(T_{p}\right) \in \mathcal{E}
\end{array}
$$

where the cost functional is given by:

$$
J\left(\bar{u}(\cdot) ; x_{i}\right):=\int_{0}^{T_{p}} F(\bar{x}(\tau), \bar{u}(\tau)) d \tau+E(\bar{x}(\bar{p})) .
$$

The bar denotes internal controller variables, $\bar{x}(\cdot)$ is the solution of (3a) driven by the input $\bar{u}(\cdot):\left[0, T_{p}\right] \rightarrow$ $\mathcal{U}$ with initial condition $x_{i}$. The stage cost to be minimized over the control horizon $T_{p}$ is given by $F(\bar{x}, \bar{u})$. We assume that $F$ satisfies:

Assumption $3 \quad F: \mathbb{R}^{n} \times \mathcal{U} \rightarrow \mathbb{R}$ is locally Lipschitz with $F(0,0)=0$ and $F(x, u)>0 \forall \mathbb{R}^{n} \times \mathcal{U} \ni(x, u) \neq$ $(0,0)$.

The solution to the optimal control problem is denoted by $\bar{u}^{\star}\left(\cdot ; x_{i}\right)$ in the following. If the optimal control problem is solved at time $t_{i}$, then the system input between two sampling instants at time $s \in\left[t_{i}, t_{i}+\delta\right)$ is given by:

$$
u\left(s ; x_{i}\right)=\bar{u}^{\star}\left(s-t_{i} ; x_{i}\right) .
$$

The constraint (3c) in the NMPC open loop optimal control problem forces the final predicted state at time $\tau=T_{P}$ to lie in a terminal region denoted by $\mathcal{E}$ and is thus often called terminal region constraint. In the cost functional $J$, the deviation from the origin of the final predicted state is penalized by the final state penalty term $E$. We further assume, that: 
Assumption 4 The system (1) has an unique continuous solution for any initial condition in the region of interest and any piecewise continuous and rightcontinuous input function $u(\cdot):\left[0, T_{p}\right] \rightarrow \mathcal{U}$.

If $T_{p}, E, F$ are chosen suitably, stability can be guaranteed. Several NMPC concepts that guarantee stability and that are similar to this setup have been proposed e.g. see (Mayne et al. 2000, Allgöwer et al. 1999) for a review. We will use the following result, which is a slight modification of Theorem 4.1 in (Chen 1997):

Theorem 3.1 Suppose

(a) that Assumptions 1-4 are satisfied,

(b) $E$ is $C^{1}, \mathcal{E} \subseteq X$ is closed and connected with the origin contained in $\mathcal{E}$ and there exists a continuous control law $k: \mathbb{R}^{n} \rightarrow \mathbb{R}^{p}$ with $k(0)=0$ such that $k(x) \in \mathcal{U} \forall x \in \mathcal{E}$ and

$$
\frac{\partial E}{\partial x} f(x, k(x))+F(x, k(x)) \leq 0, \quad \forall x \in \mathcal{E} .
$$

(c) the NMPC open-loop optimal control problem has a feasible solution for $t=0$.

Then for any sampling time $0<\delta<T_{p}$ the closedloop system is asymptotically stable with the region of attraction $\mathcal{R}$ being the set of states for which the openloop optimal control problem has a feasible solution.

Notice, that assumption (c) is, as usual in NMPC, an implicit controllability assumption. Additionally to the given assumptions, we will later need that the state feedback defined via the NMPC open loop optimal control problem and (4) is uniformly locally Lipschitz: Assumption 5 The optimal control $\bar{u}^{\star}(\tau ; x(t))$ is uniformly locally Lipschitz in $x$ in the region of attraction R

$$
\left\|\bar{u}^{\star}\left(\tau ; x_{1}\right)-\bar{u}^{\star}\left(\tau ; x_{2}\right)\right\| \leq L_{u}\left\|x_{1}-x_{2}\right\| \forall \tau \in\left[0, T_{p}\right) .
$$

In words, this (quite frequently made) assumption means that two "close" initial conditions must lead to "close" optimal input trajectories. Note that this does not exclude piecewise continuous input signals as is often used in NMPC. The assumption is rather strong, for example it excludes systems that can only be stabilized by discontinuous feedback (as state feedback NMPC can stabilize, (Fontes 2000)). From the assumptions made, we can easily deduce that the value function $V(x)=J\left(\bar{u}^{\star}(\cdot ; x) ; x\right)$ satisfies the following:

Proposition 3.1 The value function $V(x)=$ $J\left(\bar{u}^{\star}(\cdot ; x) ; x\right)$ is locally Lipschitz for all $x \in \mathcal{R}$.

To simplify the derivations, we state some properties of $V(x)$ as given in (Chen 1997):

Lemma 3.1 The value function $V(x), V: R \rightarrow \mathbb{R}$, has the following properties:

- $V(0)=0$ and $V(x)>0$ for $x \neq 0$

- Along trajectories starting from any $x\left(t_{0}\right) \in \mathcal{R}$, for $t_{0} \leq s_{1} \leq s_{2} \leq \infty$

$$
V\left(x\left(s_{2}\right)\right)-V\left(x\left(s_{1}\right)\right) \leq-\int_{s_{1}}^{s_{2}} F\left(x(\tau), u\left(\tau ; x_{i}\right)\right) d \tau
$$

holds with $u\left(s ; x_{i}\right)$ given by (4).

Later on we show recovery of performance with respect to an arbitrary compact set $\mathcal{S} \subset \mathcal{R}$. We need, that for any set $S$ we can find a compact outer approximation $\Omega(S)$ that contains $S$ and is invariant under state feedback. Thus we assume:
Assumption 6 (Blanket assumption) For all compact sets $\mathcal{S} \subset \mathcal{R}$ containing the origin, there is a constant $c>\max _{x \in \mathcal{S}} V(x)$, such that the compact set $\Omega(S)=$ $\{x \in X \mid V(x) \leq c\} \supset \mathcal{S}$.

The assumption on the existence of such a set $\Omega(S)$ for all compact sets $S \subset R$ is strong and difficult to check a priory. If this assumption is not fulfilled, the recovery of the region of attraction will be limited to sets contained in the largest level set of $V$ contained in $\mathcal{R}$. Further work will investigate in relaxing the above assumption.

Note that since $S$ is a real subset of $\mathcal{R}$ and the input is bounded, it is always possible to find a small sampling time $\tilde{\delta}$ such that for sampling times $\delta \leq \tilde{\delta}$ the state trajectory starting with initial condition $x(0) \in$ $S$, controlled during the first sampling interval with "open loop" control $u^{*}(\tau ; \hat{x}(0))$ for $\hat{x}(0) \in Q$, where $Q$ is a compact subset of $\mathbb{R}^{n}$, does not leave $\Omega(S)$.

Fact 3.1 From the compactness of $\Omega(S)$ for all $S \subset R$ and Assumption 3 it follows, that there exist finite constants $L_{F u}$ and $L_{F x}$, such that:

$$
\begin{aligned}
& \left|F\left(x_{1}, u_{1}\right)-F\left(x_{2}, u_{2}\right)\right| \leq L_{F x}\left\|x_{1}-x_{2}\right\| \\
& \quad+L_{F u}\left\|u_{1}-u_{2}\right\| \quad \forall x_{1}, x_{2} \in \Omega(S), u_{1}, u_{2} \in \mathcal{U} .
\end{aligned}
$$

\subsection{High gain observer}

The observer used is a high gain observer as in (Atassi and Khalil 1999, Teel and Praly 1995, Tornambè 1992). It is given by:

$$
\dot{\hat{x}}=A \hat{x}+B \phi(\hat{x}, u)+H(y-C \hat{x})
$$

where $H=$ blockdiag $\left[H_{1}, \ldots, H_{p}\right]$ with

$$
H_{i}^{T}=\left[\alpha_{1}^{(i)} / \varepsilon, \alpha_{2}^{(i)} / \varepsilon^{2}, \ldots, \alpha_{n}^{(i)} / \varepsilon^{r_{i}}\right] .
$$

The $\alpha_{j}^{(i)}$ s are such, that the polynomial

$$
s^{n}+\alpha_{1}^{(i)} s^{n-1}+\cdots+\alpha_{n-1}^{(i)} s+\alpha_{n}^{(i)}=0, i=1, \ldots, p
$$

is Hurwitz $\cdot \frac{1}{\varepsilon}$ is the high-gain parameter.

Remark 3.1 Notice that the use of an observer makes it necessary that the open loop input is also defined and bounded for (estimated) states that are outside of the feasiblity region of the controller. We simply define the open loop input for $\hat{x} \notin \mathcal{R}$ as fixed to an arbitrary value $u_{f} \in \mathcal{U}$ :

$$
u\left(s ; \hat{x}_{i}\right)=u_{f}, \forall \hat{x}_{i} \notin \mathcal{R}, s \in[t, t+\delta) .
$$

Note that the input constraints take care of the peaking of the observer (Esfandiari and Khalil 1992).

\section{STABILITY AND RECOVERY OF PERFORMANCE}

In this section the main results, i.e. semiglobal practical stability of the closed loop, recovery of the region of attraction and convergence of trajectories is established. We follow in principle the lines of (Atassi and Khalil 1999). In the first step we establish, that for any compact set of initial conditions of the state that is a subset of the region of attraction of the state feedback and any compact set of initial conditions of the observer the closed loop states stay bounded 
for a small enough $\varepsilon$ and enter in finite time a positively invariant region (which is arbitrarily small in the direction of the observer states). In a next step semiglobal practical stability in the sense that the trajectories come arbitrarily close to the origin (in finite time) is established. Additionally, it is shown that the trajectories of the state and output feedback controller can be made arbitrarily close for small enough $\varepsilon$ and $\delta$. The main difference to (Atassi and Khalil 1999) lies in the fact that we use a sampled NMPC scheme and thus can not fall back to standard Lyapunov and converse Lyapunov arguments. Instead decreasing properties of the value function along solution trajectories for small enough $\varepsilon$ and $\delta$ are used.

\subsection{Setup}

Define $\eta$ as the scaled observer error,

$$
\eta=\left[\eta_{11}, \ldots, \eta_{1 r_{1}}, \ldots, \eta_{p 1}, \ldots, \eta_{p r_{p}}\right], \text { with } \eta_{i j}=\frac{x_{i j}-\hat{x}_{i j}}{\varepsilon^{r_{i}-j}}
$$

Hence we have $\hat{x}=x-D(\varepsilon) \eta$ with

$$
\begin{aligned}
D(\varepsilon) & =\operatorname{blockdiag}\left[D_{1}, D_{2}, \ldots, D_{p}\right], \\
D_{i} & =\operatorname{diag}\left[\varepsilon^{r_{i}-1}, \ldots, 1\right]
\end{aligned}
$$

The closed loop system is given by

$$
\begin{aligned}
& \qquad \begin{aligned}
\dot{x}(t) & =A x(t)+B \phi\left(x(t), u\left(t ; x_{i}-D(\varepsilon) \eta_{i}\right)\right) \\
\varepsilon \dot{\eta}(t) & =A_{0} \eta(t)+\varepsilon B g\left(t, x(t), x_{i}, D(\varepsilon) \eta(t), D(\varepsilon) \eta_{i}\right)
\end{aligned} \\
& \text { with } g\left(t, x(t), x_{i}, D(\varepsilon) \eta(t), D(\varepsilon) \eta_{i}\right) \\
& =\phi\left(x(t), u\left(t, x_{i}-D(\varepsilon) \eta_{i}\right)\right)- \\
& \qquad \phi\left(x(t)-D(\varepsilon) \eta(t), u\left(t ; x_{i}-D(\varepsilon) \eta_{i}\right)\right)
\end{aligned}
$$

and $A_{0}=\varepsilon D^{-1}(\varepsilon)(A-H C) D(\varepsilon)$ where $A_{0}$ is Hurwitz. In the following the initial states are $x(0) \in \mathcal{S}$, where $S$ is any compact set in the interior of $\mathcal{R}$. The observer initial states are in $\hat{x}(0) \in Q . Q$ is any compact subset of $\mathbb{R}^{n}$.

Similar to (Atassi and Khalil 1999), for simplicity, we will write the closed loop as

$$
\dot{x}(\tau ; \varepsilon)=f_{r}\left(\tau, x(\tau ; \varepsilon), x_{i}, D(\varepsilon) \eta_{i}\right) .
$$

The trajectories of the closed loop output feedback system will be denoted $(x(\cdot ; \varepsilon), \eta(\cdot ; \varepsilon))$, starting from $(x(0), \eta(0))$. The closed loop under state feedback is denoted by $\dot{x}_{r}(\tau)=f_{r}\left(\tau, x_{r}(\tau), x_{i}, 0\right)$ with trajectories $x_{r}(\cdot)$. The resulting (sampled) open-loop control based on the observed state, will be denoted $u(\tau ; \varepsilon)$, and the control based on the real state will be denoted $u_{r}(\tau)$.

\subsection{Preliminaries}

Before we move to the main results we establish the existence of an invariant region for the observer for all system states $\Omega(S)$. Furthermore we show, that the integrated error between the state feedback and output feedback can be made sufficiently small by decreasing $\varepsilon$ and $\delta$ if the observer error starts in a sufficiently small region. This implies, that the "difference" between the "resulting" value functions can be made sufficiently small. Let $\rho=16 k_{1}^{2}\left\|P_{0}\right\|^{3}$, $W(\eta)=\eta^{T} P_{0} \eta$ and $P_{0}$ the solution of the Lyapunov equation $P_{0} A_{0}+A_{o}^{T} P_{0}=-I$. Furthermore let $k_{1}$ be a bound for the "observer error" nonlinearity for all $\eta \in \mathbb{R}^{n}$ and $x \in \Omega(S)$ :

$$
\left\|g\left(t, x(t), x_{i}, D(\varepsilon) \eta(t), D(\varepsilon) \eta_{i}\right)\right\| \leq k_{1} .
$$

Due to the assumptions made and the fact, that $\mathcal{U}$ is compact we know that such a $k_{1}$ exist. Then the following can be established:

Lemma 4.1 The set $\left\{\eta \mid W(\eta) \leq \rho \varepsilon^{2}\right\}$ is invariant for the observer error if $x \in \Omega(\mathcal{S})$.

The proof is similarly to (Atassi and Khalil 1999).

Lemma 4.2 Consider any $\xi_{1}^{\star}>0$. Then there exists an $\varepsilon_{1}^{\star}>0$ and $\delta_{1}^{\star}>0$ such that for all $0<\varepsilon \leq \varepsilon_{1}^{\star}$ and $0<\delta \leq \delta_{1}^{\star}$ for $\eta(t) \in\left\{\eta \mid W(\eta) \leq \rho \varepsilon^{2}\right\}, x(t) \in \mathcal{S}$

$\left|\int_{t}^{t+T} F(x(\tau ; \varepsilon), u(\tau ; \varepsilon)) d \tau-\int_{t}^{t+T} F\left(x_{r}(\tau), u_{r}(\tau)\right) d \tau\right| \leq T \xi_{1}^{\star}$ holds for any $T \in\left(0, T^{\star}\right]$ where $T^{\star}>\delta$ is finite, such that $x(t+\tau ; \varepsilon) \in \mathcal{R} \forall \tau \in\left(t, T^{\star}\right]$.

Lemma 4.3 Consider $T \in\left(0, T^{\star}\right]$ where $T^{\star}>\delta$ is finite, and such that $x(t+\tau ; \varepsilon) \in \mathcal{R}$ and $x_{r}(t+\tau) \in$ $\mathcal{R} \forall \tau \in\left[t, t+T^{\star}\right)$, both starting at $x(t) \in \Omega(S)$. Then for every $\xi_{2}^{\star}>0$ there exists $\varepsilon_{2}^{\star}>0$ and $\delta_{2}^{\star}>0$ such that for every $0<\varepsilon<\varepsilon_{2}^{\star}$ and $0<\delta<\delta_{2}^{\star}$ and $\eta(t) \in$ $\left\{\eta \mid W(\eta) \leq \rho \varepsilon^{2}\right\}$

$$
\left|V(x(t+T ; \varepsilon))-V\left(x_{r}(t+T)\right)\right| \leq T \xi_{2}^{\star} .
$$

\subsection{Boundedness}

Theorem 4.1 Under the Assumptions 1-5, there exists an $\varepsilon_{2}^{\star}>0$ such that, for every $0<\varepsilon \leq \varepsilon_{2}^{\star}$, the trajectories $(x(\tau ; \varepsilon), \eta(\tau))$, starting at $(x(t), \eta(t)) \in \mathcal{S} \times Q$, are bounded for all $\tau \geq t$.

Proof. Let $\Lambda=\Omega(S) \times\left\{\eta \in Q \mid W(\eta) \leq \rho \varepsilon^{2}\right\}$. We show that for small enough $\varepsilon$ and $\delta$, the compact set $\Lambda$ is positively invariant, and that trajectories starting in $S \times Q$ enter it in finite time.

Invariance of the set $\Lambda$ : Define $\partial \Omega(S)$ as the boundary of $\Omega(S)$. For $(x(t), \eta(t)) \in \partial \Omega(S) \times\left\{\eta \mid W(\eta) \leq \rho \varepsilon^{2}\right\}$ and all $T \in\left(0, T^{\star}\right]$ where $T^{\star}>\delta$ is finite, we have that

$$
\begin{aligned}
V & (x(t+T ; \varepsilon))-V(x(t)) \\
\leq & V\left(x_{r}(t+T)\right)-V(x(t)) \\
& +\left|V(x(t+T ; \varepsilon))-V\left(x_{r}(t+T)\right)\right| \\
\leq & -\int_{t}^{t+T} F\left(x_{r}(\tau), u_{r}(\tau)\right) d \tau \\
& +\left|V(x(t+T ; \varepsilon))-V\left(x_{r}(t+T)\right)\right| \\
\leq & -\int_{t}^{t+T} F(x(\tau ; \varepsilon), u(\tau ; \varepsilon)) d \tau \\
& +\left|\int_{t}^{t+T} F(x(\tau ; \varepsilon), u(\tau ; \varepsilon))-F\left(x_{r}(\tau), u_{r}(\tau)\right) d \tau\right| \\
& +\left|V(x(t+T ; \varepsilon))-V\left(x_{r}(t+T)\right)\right|
\end{aligned}
$$

We note, that since $\Omega(S) \subset R \mathcal{R} \exists T^{\star}$ such that $\forall T \leq T^{\star}$ $x(t+T ; \varepsilon) \in \mathcal{R}$, so we can apply Lemma 4.2 and Lemma 4.3

$$
\begin{aligned}
& V(x(t+T ; \varepsilon))-V(x(t)) \\
& \leq-\int_{t}^{t+T} F(x(\tau ; \varepsilon), u(\tau ; \varepsilon)) d \tau+T \xi_{1}^{\star}+T \xi_{2}^{\star}
\end{aligned}
$$

Since $x(t) \neq 0$ and $T \geq 0$ is finite, we know that

$$
\int_{t}^{t+T} F(x(\tau ; \varepsilon), u(\tau ; \varepsilon)) d \tau \geq T \kappa_{1}
$$


for some $\kappa_{1}>0$. It follows that there exists $\varepsilon_{1}^{\star}>0$, $\varepsilon_{2}^{\star}>0$ and $\delta_{1}^{\star}>0$, such that for $0<\varepsilon<\min \left(\varepsilon_{1}^{\star}, \varepsilon_{2}^{\star}\right)$ and $0<\delta<\delta_{1}^{\star}$, we have $-\kappa_{1}+\xi_{1}^{\star}+\xi_{2}^{\star} \leq 0$, and $V(x(t+T ; \varepsilon))-V(x(t)) \leq 0$. This shows that $x(t+$ $T ; \varepsilon) \in \Omega(S) \subset \mathcal{R}$, hence this holds for all $T \in\left(0, T^{\star}\right]$. If, for some time $t>T^{\star}, x(t, \varepsilon)$ should reach the border of $\Omega(S)$ again, we can reapply the above reasoning. Further, for $(x(t), \eta(t)) \in \Omega(\mathcal{S}) \times\left\{\eta \mid W(\eta)=\rho \varepsilon^{2}\right\}$ we have from Lemma 4.1 that $\dot{W} \leq 0$ if $\rho=16\left\|P_{0}\right\|^{3} k_{1}^{2}$. We conclude that for $0<\varepsilon \leq \min \left(\varepsilon_{1}^{\star}, \varepsilon_{2}^{\star}\right)$ and $0<\delta \leq$ $\delta_{1}^{\star}$, the set $\Lambda$ is positively invariant.

Finite time attractivity of $\Lambda$ : The same way as in (Atassi and Khalil 1999), consider $(x(0), \eta(0)) \in \mathcal{S} \times$ $Q$. There exists a time $T_{0}$ such that $x(\tau, \varepsilon)$ does not leave $\Omega(S)$ on the interval $\left[0, T_{0}\right]$. Also, the set $\left\{\eta \mid W(\eta) \leq \rho \varepsilon^{2}\right\}$ is reached by $\eta$ in time $T(\varepsilon)$ where $T(\varepsilon) \rightarrow 0$ as $\varepsilon \rightarrow 0$, and $\eta(t)$ is bounded on the time interval. Choose $\varepsilon_{2}$ such that for $\varepsilon \leq \varepsilon_{2}, T(\varepsilon) \leq T_{0}$. Then $(x(T(\varepsilon) ; \varepsilon), \eta(T(\varepsilon))) \in \Lambda$, and the trajectories stay bounded, since $\Lambda$ is positively invariant.

\subsection{Semiglobal Practical stability}

We show that for any small ball around the origin there exists a observer gain and a sampling time, such that trajectories will reach the ball in finite time and that the ball is invariant.

Theorem 4.2 Let the conditions of Theorem 4.1 hold. Then, for any $\xi>0$, there exists $\varepsilon_{3}^{\star}>0, \delta_{3}^{\star}>0$ and $T_{1}$ such that for every $0<\varepsilon<\varepsilon_{3}^{\star}$ and $0<\delta<\delta_{3}^{\star}$, we have $\|x(t ; \varepsilon)\|+\|\eta(t ; \varepsilon)\| \leq \xi, \forall t>T_{1}$.

Proof. Given $\xi$. As shown in (Atassi and Khalil 1999) we can find $\varepsilon_{3} \leq \varepsilon_{1}^{\star}$ such that for all $0<\varepsilon<\varepsilon_{3}$ we have $\|\eta(t ; \varepsilon)\| \leq \xi / 2, \quad \forall t \geq T\left(\varepsilon_{3}\right)$. Then, since $V(x)$ is continuous at the origin and $V(0)=0$, it follows that it is possible to find a $c_{1}$ such that $\{x \mid V(x) \leq$ $\left.c_{1}\right\} \subset\{x \mid\|x\| \leq \xi / 2\}$. With the same reasoning as in Theorem 4.1, we can show that for $0<\varepsilon<\varepsilon_{3}$ and $0<\delta<\delta_{3}$

$$
\begin{gathered}
<\delta<\delta_{3} \\
V\left(x\left(t+T^{\prime} ; \varepsilon\right)\right)-V(x(t)) \leq-\int_{t}^{t+T^{\prime}} F(x(\tau ; \varepsilon), u(\tau ; \varepsilon)) d \tau \\
+T^{\prime}\left(\xi_{1}^{\star}+\xi_{2}^{\star}\right) .
\end{gathered}
$$

Note that there exists a $\kappa_{2}>0$ such that $F(x, u) \geq \kappa_{2}$ for $x \in \Omega(\mathcal{S})-\left\{x \mid V(x) \leq c_{1}\right\}, u \in \mathcal{U}$. Assume now that $x(\tau ; \varepsilon), t \leq \tau \leq t+T^{\prime}$ is not in $\left\{x \mid V(x) \leq c_{1}\right\}$. Then, we have that

$$
V\left(x\left(t+T^{\prime} ; \varepsilon\right)\right)-V(x(t)) \leq-\kappa_{2} T^{\prime}+T^{\prime} \xi_{1}^{\star}+T^{\prime} \xi_{2}^{\star}
$$

Choose $\varepsilon$ and $\delta$ such that $\xi_{1}^{\star}+\xi_{2}^{\star}-\kappa_{2}<0$. If now $T^{\prime}$ is large enough (we can choose it as large as we want, Lemma 4.2 and 4.3 will hold since we are in the invariant region $\Lambda)$, we have that $V(x(t+$ $T ; \varepsilon)) \leq c_{1}$ for some $T \leq T^{\prime}$. Then, by contradiction, we must have $x(t+T ; \varepsilon) \in\left\{x \mid V(x) \leq c_{1}\right\}$. By the same reasoning as in Theorem 4.1, this set is invariant, and we can choose $T_{1}=\max \left(T\left(\varepsilon_{3}\right), T^{\prime}\right)$.

This establishes our main result. The closed loop system is semiglobal practically stable in the sense, that for any $S \subset R$ and any ball around the origin there exists observer gain and sampling time, such that we reach the ball from any point in $\mathcal{S}$ in finite time and that the ball is positively invariant.

Further we can show, that the trajectories of the output feedback converges, uniformly in $t$, to the trajectories of the state feedback as $\varepsilon \rightarrow 0, \delta \rightarrow 0$ :
Theorem 4.3 Given any $\xi>0$. Under the conditions in Theorem 4.1, there exists $\varepsilon_{4}^{\star}$ and a $\delta_{4}^{\star}$ such that for every $0<\varepsilon \leq \varepsilon_{4}^{\star}$ and $0<\delta \leq \delta_{4}^{\star}\left\|x(t ; \varepsilon)-x_{r}(t)\right\| \leq$ $\xi, \forall t \geq 0$.

Proof. Follows from Theorem 4.2, on the interval $\left[T_{1}, \infty\right)$, and the same on the (peaking) interval $[0, T(\varepsilon)]$. On the interval $\left[T(\varepsilon), T_{1}\right]$ it follows from the continuous dependence of solutions of differential equations on parameters and initial conditions over compact time intervals. See (Atassi and Khalil 1999) for further details.

\section{EXAMPLE: PENDULUM ON A CART}

We examplify the approach considering the control of an inverted pendulum on a car, as shown in Fig. 5. The angle of the pendulum is measured and denoted

Fig. 1. Pendulum on a cart

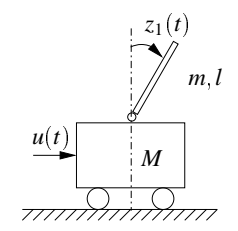

in the following by $z_{1}$. The input to the system is given by $u$ which acts on the cart's translation and is limited to $-10 \leq u(t) \leq 10$. The control objective is to stabilize the angle $z_{1}=0$ (upright position) while the cart's position is not limited. The resulting model is of second order and fits the considered system class (Imsland et al. 2001a). As NMPC control scheme, the so called quasi-infinite horizon NMPC scheme (Chen and Allgöwer 1998) is used. The choice of the terminal region/terminal penalty and the other controller parameters are given in (Imsland et al. 2001a). We show the recovery of the region of attraction and the recovery of performance for a fixed sampling time $\delta=0.05 \mathrm{~s}$. Figure 5 shows a part of the region of attraction for different values of $\varepsilon=0.03$, 0.07 and 0.09 in comparison to the state feedback case. As can be seen, the region of attraction of the output-feedback controller converges to the state feedback for decreasing $\varepsilon$. This is in correspondence to the result of Theorem 4.2. Figure 5 shows the closed

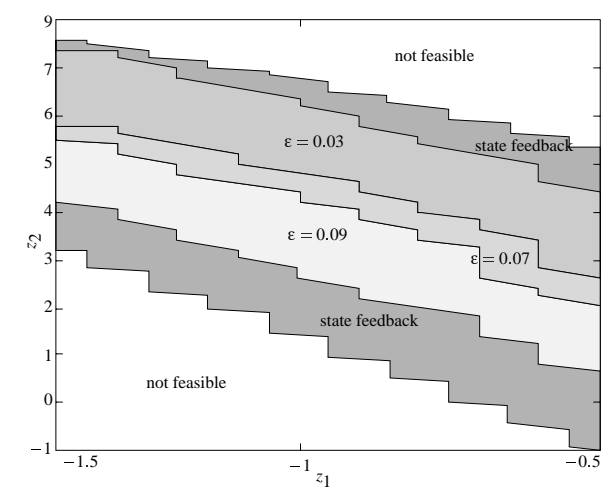

Fig. 2. Recovery of region of attraction.

loop pendulum angle $z_{1}$ and the applied input $u$ for an initial disturbance for $\varepsilon=0.03,0.09$ in comparison to the state feedback case. The observer was initialized with a zero initial estimate of the states. As for the region of attraction, the performance is recovered for 

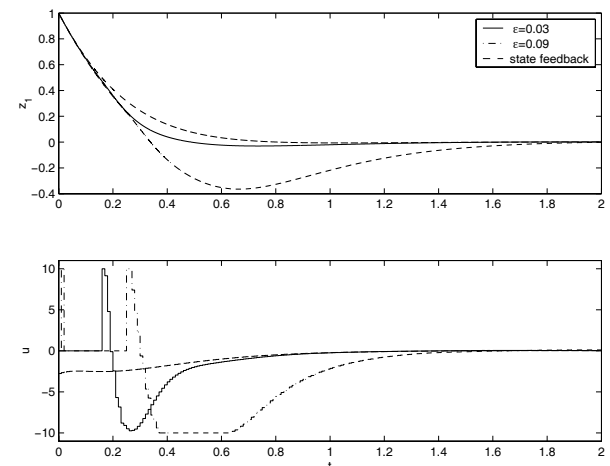

Fig. 3. Recovery of the performance.

decreasing values of epsilon. Notice also that for the shown initial conditions in the state feedback case the input constraints are not hit, while for the output feedback the NMPC controller hits the input constraints. This is due to the "observer" peaking in the initial phase. The presented results underpin the stability and performance properties derived in Section 4.

\section{CONCLUSIONS}

In recent years significant progress in the area of nonlinear model predictive control has been made. With respect to the output feedback case, however no significant progress has been made so far. The existing solutions are either of local nature (Scokaert et al. 1997, Magni et al. 1998, Magni et al. 2001) or difficult to implement (Michalska and Mayne 1995). In this paper we have shown, that using a high-gain observer and a "sampled" NMPC controller for a special system class semiglobal practical stability and recovery of performance for the closed loop can be achieved. The results are valid for a wide class of stabilizing NMPC schemes. Thus they can be considered as a special separation principle for NMPC. However there remain a couple of open question and problems. For example the properness assumption on the value function of the state feedback controller in the region of attraction are difficult to check and limits the applicability. Also the class of systems the approach is applicable to is limited. Further work will investigate these problems and try to overcome them as well as consider the question of asymptotic stability of the closed loop.

\section{ACKNOWLEDGEMENT}

The authors gratefully acknowledge Eric Bullinger from the IST, University of Stuttgart for many helpful discussions and Tobias Pfisterer from the University of Stuttgart for helping with the preparation of the simulations.

\section{REFERENCES}

Allgöwer, F., T. A. Badgwell, J. S. Qin, J. B. Rawlings and S. J. Wright (1999). Nonlinear predictive control and moving horizon estimation An introductory overview. In: Advances in Control, Highlights of ECC'99 (P. M. Frank, Ed.). pp. 391-449. Springer.

Atassi, A.N. and H.K. Khalil (1999). A separation principle for the stabilization of a class of nonlinear systems. IEEE Trans. Automatic Control 44(9), 1672-1687.

Chen, H. (1997). Stability and Robustness Considerations in Nonlinear Model Predictive Control. Fortschr.-Ber. VDI Reihe 8 Nr. 674. VDI Verlag. Düsseldorf.
Chen, H. and F. Allgöwer (1998). A quasi-infinite horizon nonlinear model predictive control scheme with guaranteed stability. Automatica 34(10), 1205-1218.

Esfandiari, F. and H.K. Khalil (1992). Output feedback stabilization of fully linearizable systems. Int. J. Control 56(5), 1007-1037.

Fontes, F.A. (2000). A general framework to design stabilizing nonlinear model predictive controllers. Syst. Contr. Lett. 42(2), 127-143.

Imsland, L., R. Findeisen, E. Bullinger, F. Allgöwer and B.A. Foss (2001a). A note on stability, robustness and performance of output feedback nonlinear model predictive control.. Accepted for publication in J. Proc. Contr.

Imsland, L., R. Findeisen, E. Bullinger, F. Allgöwer and B.A. Foss $(2001 b)$. On output feedback nonlinear model predictive control using high gain observers for a class of systems. In: 6th IFAC Symposium on Dynamics and Control of Process Systems, DYCOPS-6. Jejudo, Korea. pp. 91-96.

Isidori, A. (1995). Nonlinear Control Systems. 3. ed.. Springer-Verlag. Berlin.

Maggiore, M. and K. Passino (2000). Output feedback control of stabilizable and incomplete observable systems: Theory. In: Proc. Amer. Contr. Conf.. ACC. Chicago. pp. 3641-3645.

Magni, L., D. De Nicolao and R. Scattolini (1998). Output feedback receding-horizon control of discrete-time nonlinear systems. In: Preprints of the 4th Nonlinear Control Systems Design Symposium 1998 - NOLCOS'98. IFAC. pp. 422-427.

Magni, L., G. De Nicolao and R Scattolini (2001). Output feedback and tracking of nonlinear systems with model predictive control. Automatica 37(10), 1601-1607.

Mayne, D.Q., J.B. Rawlings, C.V. Rao and P.O.M. Scokaert (2000). Constrained model predictive control: stability and optimality. Automatica 26(6), 789-814.

Michalska, H. and D.Q. Mayne (1993). Robust receding horizon control of constrained nonlinear systems. IEEE Trans. Automat. Contr. AC38(11), 1623-1633.

Michalska, H. and D.Q. Mayne (1995). Moving horizon observers and observer-based control. IEEE Trans. Automat. Contr. 40(6), 995-1006.

Scokaert, P.O.M., J.B. Rawlings and E.S. Meadows (1997). Discrete-time stability with perturbations: Application to model predictive control. Automatica 33(3), 463-470.

Shim, H. and A.R. Teel (2001). Further results on the nonlinear separation principle: the general 'asymptotically controllable' case. In: Nonlinear Control Systems Design Symposium 2001 - NOLCOS'01. IFAC.

Teel, A. and L. Praly (1994). Global stabilizability and observability imply semi-global stabilizability by output feedback. Syst. Contr. Lett. 22(4), 313325.

Teel, A. and L. Praly (1995). Tools for semiglobal stabilization by partial state and output feedback. SIAM J. Control and Optimization 33(5), 14431488.

Tornambè, A. (1992). High-gain observers for nonlinear systems. Int. J. of Systems Science 23(9), 1475-1489. 